\title{
Associação entre hábitos alimentares e de vida de idosos hipertensos usuários de uma farmácia municipal
}

\author{
Association between eating and living habits of elderly hypertensive users of a municipal \\ pharmacy
}

\author{
Letícia Lopes Vieira ${ }^{1}$ \\ Orcid: https://orcid.org/0000-0001-9410-8044 \\ Luiza Veloso Dutra ${ }^{3}$ \\ Orcid: https://orcid.org/0000-0002-8351-655X
}

Orcid: https://orcid.org/0000-0001-9259-2055

\author{
Tânia Aparecida de Araujo ${ }^{4}$ \\ Orcid: https://orcid.org/0000-0001-5894-8695
}

\author{
Isabela Martins Oliveira ${ }^{5}$ \\ Orcid: https://orcid.org/0000-0001-5838-6944
}

\begin{abstract}
Resumo
O estado nutricional dos idosos tem um papel importante para caracterizar a qualidade de vida e a saúde destes que possuem maior tendência aàs alterações fisiológicas e sociais, doenças crônicas como hipertensão arterial, uso de medicamentos, dificuldades com a alimentação e depressão. Objetivo: analisar a associação entre hábitos alimentares e de vida de idosos hipertensos usuários de uma farmácia municipal. Metodologia: Os dados foram coletados, em uma farmácia pública do município de Visconde do Rio Branco-MG, com uma amostra de 180 idosos. Foi aplicado o questionário de frequência alimentar (QFA) para identificar os hábitos alimentares e o questionário de caracterização da idade, sexo, atividade física, hábito de fumar e renda. Os dados foram registrados em planilha eletrônica e submetidos à análise estatística no Software Stata. Resultados: 73,8\% (n=134) dos idosos eram do sexo feminino, 55,6\% $(\mathrm{n}=100)$ de 60 a 65 anos de idade, $88,3 \%$ $(\mathrm{n}=159)$ possuem renda mensal de um salário-mínimo e $64,4 \%(\mathrm{n}=116)$ nunca fizeram atividade física. Observou-se quanto à frequência do consumo alimentar que $50,3 \%$ são alimentos in natura, $14,3 \%$ ingredientes culinários, $7,1 \%$ de processados e $28,3 \%$ de ultraprocessados. Conclusões: os hábitos alimentares dos idosos não estão saudáveis, pois foi verificado predomínio de alimentos que não contribuem para a melhora do quadro da hipertensão. Ademais, aqueles que fumam consomem menos alimentos in natura e mais ultraprocessados e tomam mais medicamentos do que aqueles que não fumam. Sugere-se um acompanhamento nutricional envolto por políticas públicas que visem melhorar a qualidade de vida dessa população.
\end{abstract}

Palavras-chave: Envelhecimento; Alterações fisiológicas; Hipertensão arterial.

\begin{abstract}
The nutritional status of the elderly plays an important role in characterizing the quality of life and health of those who are more prone to physiological and social changes, chronic diseases such as high blood pressure, use of medication, difficulties with eating and depression. Objective: to analyze the association between eating habits and life habits of elderly hypertensive users of a municipal pharmacy. Methodology: Data were collected in a
\end{abstract}

\footnotetext{
${ }^{1}$ Graduanda do curso de Nutrição no Centro Universitário de Viçosa, Viçosa, Minas Gerais, Brasil. E-mail: leticia-lopesvieira@hotmail.com ${ }^{2}$ Graduanda do curso de Nutrição no Centro Universitário de Viçosa, Viçosa, Minas Gerais, Brasil. E-mail:

leuaniarcanjodefreitas@gmail.com

${ }^{3}$ Docente no curso de Nutrição no Centro Universitário de Viçosa, Viçosa, Minas Gerais, Brasil. E-mail: luizavdutra@gmail.com

${ }^{4}$ Docente do curso de Nutrição da Universidade Federal de Uberlândia, Uberlândia, Minas Gerais, Brasil. E-mail:

tanniaraujo@hotmail.com.br

${ }^{5}$ Doutoranda em Saúde Pública da Universidade São Paulo, USP, São Paulo, Brasil. E-mail: isabelamgo@gmail.com
} 
public pharmacy in the city of Visconde do Rio Branco-MG, with a sample of 180 elderly people. The food frequency questionnaire (FFQ) was applied to identify eating habits and the questionnaire to characterize age, sex, physical activity, smoking and income. Data were registered in an electronic spreadsheet and submitted to statistical analysis in the Stata Software. Results: $73.8 \%(\mathrm{n}=134)$ of the elderly were female, $55.6 \%(\mathrm{n}=100)$ from 60 to 65 years of age, $88.3 \%(n=159)$ have a monthly income of one salary minimum and $64.4 \%$ $(\mathrm{n}=116)$ had never done physical activity. It was observed as to the frequency of food consumption that $50.3 \%$ are fresh food, $14.3 \%$ culinary ingredients, $7.1 \%$ processed and $28.3 \%$ ultra-processed. Conclusions: the eating habits of the elderly are not healthy, as there was a predominance of foods that do not contribute to the improvement of hypertension. Furthermore, those who smoke consume less fresh and more ultra-processed foods and take more medications than those who do not smoke. It is suggested a nutritional follow-up surrounded by public policies that aim to improve the quality of life of this population.

Keywords: Aging; Physiological changes; Arterial hypertension.

\section{Introdução}

O envelhecimento dos seres humanos é um processo natural que cursa com progressivas alterações fisiológicas ${ }^{1}$. Essas alterações, por sua vez, podem ser sensoriais, como a visão, o tato, paladar e o cheiro, bem como gastrintestinais, hepáticas e cardíacas. Mudanças que podem levar a distúrbios nutricionais, como a hipertensão, obesidade e desnutrição que comprometem, gradativamente, o estado nutricional dos idosos $^{2}$.

A qualidade de vida pode estar associada à satisfação pessoal, valores e cultura de um indivíduo. Além da capacidade funcional, do nível socioeconômico e do estado emocional, entre outros ${ }^{3}$. Nesse contexto, assim como em outras fases da vida, a velhice é um estado relacionado com a saúde e marcada por percepções que podem impactar, de maneira positiva ou negativa, na qualidade de vida do indivíduo ${ }^{34}$.

$\mathrm{O}$ estado nutricional do idoso tem um papel importante para caracterizar a qualidade de vida (QV) e a saúde dessa população ${ }^{3}$. A desnutrição é um fator relevante nos idosos, pois está relacionada ao aumento da incapacidade funcional, aumento do número de internações, grande facilidade de adquirir infecções e diminuição da qualidade de vida ${ }^{5}$. Já a obesidade é uma doença crônica não transmissível (DCNT) que acarreta várias outras patologias, como: doenças cardiovasculares, câncer, diabetes mellitus, hipertensão arterial, o que vai prejudicar o perfil de morbimortalidades das populações, bem como o uso de medicamentos 6 .

A adoção de hábitos alimentares saudáveis (como a redução de gorduras e açúcares e ingestão de frutas e verduras) faz parte das recomendações da Organização Mundial da Saúde (OMS) no tratamento não farmacológico da hipertensão. A OMS conscientiza, ainda, sobre adoção de hábitos alimentares saudáveis (maior consumo de frutas e hortaliças e menor consumo de sódio, açucares e gorduras saturadas) como um importante meio de controlar as DCNT e suas complicações secundárias à saúde ${ }^{7}$.

O Guia Alimentar para a População Brasileira foi criado com a finalidade de apresentar para os brasileiros as diretrizes oficiais no que se refere à alimentação. Esse material dispõe os grupos de alimentos: in natura, minimamente processados, ingredientes culinários, processados e ultraprocessados. Além disso, aborda sobre alimentação saudável, a qual deve ainda ir além dos aspectos biológicos e sociais do indivíduo, devem estar em consonância com a cultura alimentar, as dimensões de gênero, raça e etnia. Sendo acessível, físico e financeiramente, em harmonia com a quantidade e qualidade, eficientes na variedade, equilíbrio, moderação e prazer ${ }^{8}$.

Entende-se que a investigação da avaliação dos hábitos alimentares de idosos hipertensos, sob o olhar das alterações 
fisiológicas, como a hipertensão, pode subsidiar ajustes nas políticas públicas de prevenção e promoção de saúde, a partir da compreensão destes fatores intervenientes nos agravos nutricionais. Diante disso, o presente trabalho tem por objetivo analisar a associação entre hábitos alimentares e de vida de idosos hipertensos usuários de uma farmácia municipal.

\section{Materiais e Métodos}

Trata-se de um estudo observacional transversal, realizado com idosos (60 anos ou mais) hipertensos atendidos pela Farmácia Municipal de Visconde do Rio Branco, Minas Gerais. Como critério de exclusão, não participaram da pesquisa os idosos normotensos, com algum tipo de declínio cognitivo (ou limitações físicas e/ou cognitivas para responder ao questionário), não usuários da Farmácia Municipal e não residentes no município de Visconde do Rio Branco - MG.

Para o cálculo da amostra considerou-se o número de idosos hipertensos cadastrados na Farmácia Municipal de Visconde do Rio Branco $(n=800)$ e um erro tolerável de $10 \%$, conforme proposto por Barbetta $(2005)^{9}$, que resultou em uma amostra de 160 idosos.

O projeto foi aprovado pelo Comitê de Ética em Pesquisas com Seres Humanos Sylvio Miguel (CEP), atendendo à Resolução 466/2012 do Conselho Nacional de Saúde, que normatiza as pesquisas envolvendo seres humanos. A coletas de dados só foram iniciadas após aprovação do CEP (3.770.464). A coleta dos dados foi realizada do final do mês de dezembro, janeiro e início de fevereiro, de segunda à sexta-feira, na Farmácia Municipal de Visconde do Rio Branco, no ano de 2019. Os participantes foram convidados na farmácia no mês de novembro, e os que aceitaram participar da pesquisa assinaram o Termo de Consentimento Livre e Esclarecido (TCLE), esclarecendo todos os riscos e benefícios da pesquisa e foram entrevistados individualmente.

Após aceite em participar da pesquisa, foi aplicado um questionário a cada idoso. Após da coleta de dados, foi realizada uma orientação para os idosos por meio de folder explicativo sobre a importância de priorizar uma alimentação rica em frutas, hortaliças e verduras, e um menor consumo de sódio (substituições do sal por temperos saudáveis), açucares, gorduras saturadas, para auxílio no controle da Hipertensão Arterial Sistêmica (HAS).

\section{Variáveis}

Características sociodemográficas descreveram a amostra e hábitos de vida foram investigados como prática de atividade física e fumo.

Os hábitos alimentares foram pesquisados por meio de questionário de frequência do consumo alimentar (QFCA) qualitativo, contendo 93 itens com 8 opções de consumo: nunca, raramente, mensal, quinzenal, 1 ou 2 vezes por semana, 3 ou 4 vezes por semana, 5 ou 6 vezes por semana, 1 vez por dia e 2 ou mais vezes por dia ${ }^{10}$.

\section{Variáveis dependentes}

As variáveis dependentes foram frequência de consumo de acordo com o grupo de alimentos por grau de processamento e quantidade de medicamentos.

Os alimentos presentes no questionário de frequência alimentar (QFA) foram classificados em grupos de acordo com o Guia Alimentar para a População Brasileira. Sendo estes: alimentos in natura e minimamente processados, ingredientes culinários, alimentos processados e alimentos ultraprocessados. Para realizar a interpretação do QFCA, foi utilizada a planilha eletrônica Microsoft Office Excel, classificando-os em 8 opções de consumo: uma vez ao dia, duas vezes ao dia, 3 a 4 vezes na semana. 
Para avaliar os hábitos alimentares, foi comparado o consumo alimentar dos idosos hipertensos, a partir da resposta ao questionário na frequência alimentar, de acordo com o grau de processamento dos alimentos (in natura, minimamente processados, processados e ultraprocessados). Esse resultado foi comparado com outras variáveis, verificando se havia relações entre $o$ consumo alimentar com hábitos de vida, bem como socioeconômicos.

A quantidade de medicamentos anti-hipertensivos que os idosos relataram utilizar foram contabilizados e descritos em grupamentos de uma a três, quatro, cinco e seis, sendo eles Atenolol, Anmlodipina, Losartana, Enalapril, Selozok e Hidroclorotiazida, que são fornecidos gratuitamente pelo Sistema Único de Saúde.

\section{Variáveis independentes}

As características sociodemográficas foram: sexo, idade (60 a 65,66 a 70, 71 a 75 e $>75$ anos) e renda mensal (1, 2 a 3, >4 salários-mínimos). E os hábitos de vida foram avaliados como: a frequência de prática de atividade física (nunca, 1 vez ou 3 vezes ou mais na semana) e o hábito de fumar ( $\operatorname{sim}$ ou não).

\section{Análise estatística}

Os dados foram organizados em planilha eletrônica e, posteriormente, analisadas com auxílio do Microsoft Office
Excel e foi feita uma tabela de frequências relativas e absolutas com as descrições dos dados quantitativos obtidos. Os dados do QFA foram analisados de forma qualitativa, por grupos de alimentos e quantitativa por frequência de consumo.

Foi realizada, inicialmente, uma análise de normalidade das variáveis por Kolmogorv Sirminov ${ }^{11}$ e constatou-se que eram não paramétricas e após uma análise descritiva dos dados por meio de cálculo de média e desvio-padrão foi utilizado o teste Mann-Whitney ${ }^{12}$ e Anova ${ }^{13}$ para as variáveis quantitativas. Os dados foram armazenados no programa Microsoft Excel e analisados no Software Stata MP 13.0 para Windows. Para as diferenças, essas foram consideradas significativas quando $\mathrm{p}<0,05$.

\section{Resultados}

Participaram do estudo 180 idosos dos quais $73,8 \%(n=134)$ eram do sexo feminino, $55,6 \%$ de 60 a 65 anos de idade $(n=100)$ e $88,3 \%(n=159)$ possuem renda mensal de um salário-mínimo. Fazem uso do medicamento atenolol 77,2\% ( $\mathrm{n}=139)$, $67,2 \%$ usam anlodipino $(\mathrm{n}=121), 95,5 \%$ losartana $\quad(\mathrm{n}=172), \quad 78,3 \% \quad$ enalapril $(\mathrm{n}=141), 30 \%$ selozok $(\mathrm{n}=54)$ e $91,1 \%$ hidroclorotiazida $\quad(n=164)$. Quanto à atividade física, $64,4 \%$ nunca fizeram $(\mathrm{n}=116)$ e $18,8 \%$ fazem atividade 1 vez por semana $(n=34)$ e $71,1 \% \quad(n=128) \quad$ dos entrevistados não fumam (Tabela 1).

Tabela 1 - Caracterização da amostra de idosos hipertensos usuários de uma farmácia municipal, Visconde do Rio Branco, Minas Gerais, 2019.

\begin{tabular}{lcc}
\hline Variáveis & n & \% \\
\hline Sexo & 133 & 73,8 \\
Feminino & 47 & 26,2 \\
Masculino & & \\
Idade & 100 & 55,6 \\
60 a 65 & 58 & 32,2 \\
66 a 70 & 10 & 5,6 \\
71 a 75 & 12 & 6,7 \\
$>75$ & & \\
Atividade física & 116 & 64,4 \\
Nunca & & \\
\hline
\end{tabular}




\begin{tabular}{lcc}
\hline 1x semana & 34 & 18,8 \\
3x ou mais na semana & 30 & 16,6 \\
Hábito de fumar & & \\
Sim & 52 & 28,8 \\
Não & 128 & 71,1 \\
Renda & & \\
1 salário mínimo & 159 & 88,3 \\
2 a salários mínimos & 21 & 11,6 \\
> 4 salários & - & - \\
Medicamentos & & \\
Atenolol & 139 & 77,2 \\
Anlodipino & 121 & 67,2 \\
Losartana & 172 & 95,5 \\
Enalapril & 141 & 78,3 \\
Selozok & 54 & 30,0 \\
Hidroclorotiazida & 164 & 91,1 \\
\hline
\end{tabular}

Observou-se quanto à frequência do consumo alimentar dos idosos que 50,3\% são alimentos in natura, $14,3 \%$ ingredientes culinários, $7,1 \%$ de processados e $28,3 \%$ de ultraprocessados. E. de acordo com os dados da Tabela 2, quando analisada a associação entre a idade e o grau de processamento dos alimentos, verificou-se que o consumo de alimentos ultraprocessados foi menor entre as faixas etárias de 60 a 65 anos e 71 a 75 anos. Os idosos que realizavam atividade física de 1 a 3 vezes ou mais na semana, consumiam mais alimentos in natura $\mathrm{e}$ aqueles que nunca praticam atividade física, consumiam mais ultraprocessados comparado aos demais grupos. Ao analisar a associação entre o grau de processamento dos alimentos e o hábito de fumar ou não, foi verificado que os idosos que fumam consomem menos alimentos in natura e mais ultraprocessados.

Tabela 2 - Frequência do consumo de alimentos por grau de processamento, de acordo com o sexo, idade, atividade física, hábito de fumar e renda de idosos, Visconde do Rio Branco, Minas Gerais, 2019.

\begin{tabular}{lccccc}
\hline Variável & $\mathbf{n}(\mathbf{\%})$ & In Natura & $\begin{array}{c}\text { Ingredientes } \\
\text { culinários }\end{array}$ & Processados & Ultraprocessados \\
\hline Sexo & & & & & \\
$\quad$ Feminino & $159(88,3)$ & $49,8 \pm 0,4$ & $14,5 \pm 0,1$ & $7,0 \pm 0,2$ & $28,7 \pm 0,4$ \\
$\quad$ Masculino & $21(11,6)$ & $51,5 \pm 1,9$ & $13,9 \pm 0,8$ & $7,9 \pm 0,7$ & $26,7 \pm 1,4$ \\
Idade & & & & & \\
60 a 65 anos & $100(55,5)$ & $49,8 \pm 0,5$ & $14,4 \pm 0,1$ & $6,8 \pm 0,2^{*}$ & $28,9 \pm 0,5$ \\
66 a 70 anos & $58(32,2)$ & $50,5 \pm 0,9$ & $14,4 \pm 0,3$ & $7,5 \pm 0,4$ & $27,6 \pm 0,7$ \\
71 a 75 anos & $10(5,6)$ & $50,8 \pm 0,9$ & $14,9 \pm 0,3$ & $6,0 \pm 0,7 *$ & $28,3 \pm 1,2$ \\
$>75$ anos & $12(6,7)$ & $48,3 \pm 1,4$ & $14,4 \pm 0,3$ & $9,0 \pm 0,9$ & $28,3 \pm 1,0$ \\
Atividade física & & & & & \\
$\quad$ Nunca & $116(64,4)$ & $48,9 \pm 0,4 *$ & $14,6 \pm 0,1$ & $7,3 \pm 0,3$ & $29,2 \pm 0,4 *$ \\
$\quad$ 1x semana & $34(18,8)$ & $52,8 \pm 1,0$ & $14,0 \pm 0,2$ & $6,5 \pm 0,4$ & $26,7 \pm 0,8$ \\
$\quad$ 3x ou mais na & $30(16,6)$ & $50,9 \pm 1,4$ & $14,4 \pm 0,5$ & $7,1 \pm 0,6$ & $27,5 \pm 0,9$ \\
semana & & & & \\
Hábito de fumar & & & & \\
\hline
\end{tabular}




\begin{tabular}{lccccc}
\hline Sim & $52(28,8)$ & $48,4 \pm 0,7^{* *}$ & $14,9 \pm 0,3^{* *}$ & $7,1 \pm 0,41$ & $29,6 \pm 0,7^{* *}$ \\
Não & $128(71,1)$ & $50,6 \pm 0,5$ & $14,3 \pm 0,2$ & $7,1 \pm 0,24$ & $28,0 \pm 0,4$ \\
Renda & & & & & \\
$\begin{array}{l}1 \text { salário-mínimo } \\
\text { 2 a 3 salários- } \\
\text { mínimos }\end{array}$ & $159(88,3)$ & $49,8 \pm 0,4$ & $14,5 \pm 0,11$ & $7,0 \pm 0,21$ & $28,7 \pm 0,4$ \\
\hline
\end{tabular}

Legenda: * diferença significante $(\mathrm{p}>0,05)$

$\mathrm{Na}$ análise de associação entre sexo e a quantidade de medicamentos não houve significância, embora tenha sido identificado que as mulheres idosas tomam, em média, mais medicamentos que os homens. Ao analisar atividade física e quantidade de medicamentos, aqueles que mais praticam atividade física consomem um menor número $(3,3 \%$ tomam 1 medicamento) e os indivíduos que fumam, tomam mais medicamentos do que aqueles que não fumam $(19,2 \%$ tomam 6 medicamentos) (Tabela 3).

Tabela 3 - Quantidade de medicamentos consumidos de acordo com o sexo, idade, atividade física, hábito de fumar e renda de idosos, Visconde do Rio Branco, Minas Gerais, 2019.

\begin{tabular}{|c|c|c|c|c|c|}
\hline \multirow{2}{*}{ Variável } & \multirow{2}{*}{ n $(\%)$} & \multicolumn{4}{|c|}{ Quantidade de medicamentos anti-hipertensivos } \\
\hline & & Um a três & Quatro & Cinco & Seis \\
\hline \multicolumn{6}{|l|}{ Sexo } \\
\hline Feminino & $134(74,5)$ & $23(17,2 \%)$ & $48(35,8 \%)$ & $53(39,5 \%)$ & $10(7,5 \%)$ \\
\hline Masculino & $46(25,5)$ & $5(10,9 \%)$ & $17(37,0 \%)$ & $16(34,8 \%)$ & $8(17,4)$ \\
\hline \multicolumn{6}{|l|}{ Idade } \\
\hline 60 a 65 anos & $100(55,5)$ & $19(19,0 \%)$ & $34(34,0 \%)$ & $35(35,0 \%)$ & $12(12,0 \%)$ \\
\hline 66 a 70 anos & $58(32,2)$ & $8(13,8 \%)$ & $20(34,5 \%)$ & $25(43,1 \%)$ & $5(8,6 \%)$ \\
\hline 71 a 75 anos & $10(5,6)$ & - & $5(50,0 \%)$ & $4(40,0 \%)$ & $1(10,0 \%)$ \\
\hline$>75$ anos & $12(6,7)$ & $1(8,3 \%)$ & $6(50,0 \%)$ & $5(41,7 \%)$ & - \\
\hline \multicolumn{6}{|l|}{ Atividade Física } \\
\hline Nunca & $116(64,4)$ & $17(14,7 \%)$ & $47(40,5 \%)$ & $38(32,8 \%)$ & $14(12,0 \%)$ \\
\hline 1x semana & $34(18,8)$ & $6(17,65 \%)$ & $7(20,6 \%)$ & $18(52,9 \%)$ & $3(8,8 \%)$ \\
\hline $3 \mathrm{x}$ ou mais na semana & $30(16,6)$ & $5(16,7 \%)$ & $11(36,7 \%)$ & $13(43,3 \%)$ & $1(3,3 \%)$ \\
\hline \multicolumn{6}{|l|}{ Hábito de fumar } \\
\hline Sim & $52(28,8)$ & $7(13,5 \%)$ & $17(32,7 \%)$ & $18(34,6 \%)$ & $10(19,2 \%)$ \\
\hline Não & $128(71,1)$ & $21(16,41 \%)$ & $48(37,5 \%)$ & $51(39,8 \%)$ & $8(6,3 \%)$ \\
\hline \multicolumn{6}{|l|}{ Renda } \\
\hline 1 salário-mínimo & $159(88,3)$ & $23(14,5 \%)$ & $57(35,9 \%)$ & $61(38,4 \%)$ & $18(11,3 \%)$ \\
\hline 2 a 3 salários-mínimos & $21(11,6)$ & $5(23,8 \%)$ & $8(38,1 \%)$ & $8(38,1 \%)$ & - \\
\hline \multicolumn{6}{|l|}{ Grupo de alimentos } \\
\hline In natura & $67,9 \pm 9,5$ & $47,9 \pm 5,6$ & $49,5 \pm 1,3$ & $50,1 \pm 0,5$ & $49,8 \pm 0,5$ \\
\hline Ingredientes & $6,1 \pm 2,4$ & $9,3 \pm 5,7$ & $7,2 \pm 0,5$ & $7,4 \pm 0,4$ & $6,7 \pm 0,3$ \\
\hline Processados & $18,6 \pm 7,0$ & $30,1 \pm 10,7$ & $28,7 \pm 1,0$ & $28,3 \pm 0,6$ & $28,8 \pm 05$ \\
\hline
\end{tabular}




\section{Discussão}

Os resultados encontrados no presente estudo apontam para aspectos relevantes ao sinalizar que os hábitos alimentares dos pacientes usuários de uma farmácia municipal não estão saudáveis pois, embora consumam alimentos como frutas, verduras e legumes, tais alimentos não fazem parte da dieta habitual desses pacientes, predominando alimentos que não contribuem para a melhora do quadro da hipertensão. Além disso, é observado o alto consumo de alimentos ricos em carboidratos simples, açúcares e gorduras saturadas.

Verificou-se a maior prevalência de idosos jovens (60 a 65 anos), do sexo feminino, com renda mensal de um saláriomínimo, que não tem o hábito de fumar e que nunca ou raramente praticam atividades físicas. As idosas apresentaram um maior uso de medicamentos. E, como esperado, os idosos mais velhos (>75 anos), de ambos os sexos, fazem uso de mais medicamentos e consomem mais alimentos ultraprocessados. Dessa forma, ainda sendo escassos os estudos que avaliam a qualidade da dieta de idosos, um estudo identificou diversos fatores relacionados à maior vulnerabilidade para o consumo de uma dieta de baixa qualidade, destacando-se as refeições com menor frequência $e$ as dificuldades funcionais para comprar ou preparar alimentos ${ }^{14}$.

Em relação à hipertensão arterial, estudos presentes na literatura, que investigaram conhecimento, atitudes e práticas de indivíduos portadores de hipertensão arterial revelam que nem sempre o conhecimento sobre a hipertensão arterial está relacionado a mudanças comportamentais ${ }^{15}$. E, com aumento de informação sobre a doença, sua forma de controle está relacionada à criação de ambientes favoráveis à saúde em nível socioeconômico, familiar e comunitário, a qual está diretamente ligada à promoção de saúde ${ }^{16}$.

São apontados para exemplificar e justificar as mudanças comportamentais, a evolução silenciosa e a natureza crônica da hipertensão. A existência de estilos de vida com atitudes, crenças, hábitos comuns a todos e condições sociais que tendem a ser estáveis através do tempo, o prazer individual por comportamentos agradáveis como tabagismo, acesso a alimentos em abundância, consumo de comida mais salgada e o sedentarismo são um poderoso impedimento para adoção de comportamentos saudáveis ${ }^{17}$.

E em relação aos entrevistados com renda de um salário mínimo é possível concluir que eles fazem o uso de mais medicamentos quando comparado aos idosos que recebem de 2 a 3 salários mínimos e mais de $60 \%$ deles não praticam atividade física. Foi observado um elevado número de uso de medicamentos entre idosos que fumam, esse fato pode dificultar o controle da HAS, podendo levar a um aumento da pressão arterial, logo, usam-se mais medicamentos ${ }^{18}$.

Há determinantes sociais e ambientais que justificam as escolhas dos pacientes idosos pela não adesão ao tratamento da HAS. Esses determinantes podem ser explicados pelas dificuldades no acesso aos serviços de saúde, analfabetismo, distúrbios de memória, dificuldade em lembrar-se do medicamento, uso de muitos medicamentos ao mesmo tempo e alto custo quando não encontrados gratuitamente, entre outros ${ }^{14}$.

O hábito de um indivíduo ou grupo também é gerado através de condições socialmente determinadas. Contudo, é dito que da mesma forma que um hábito explica os comportamentos regulares, este também é produtor da improvisação, permitindo a adaptação a uma nova realidade social, quando são feitas orientações nutricionais para as escolhas 
alimentares. $\mathrm{O}$ indivíduo tem consigo estruturas construídas interiormente que lhe permitem exteriorizar práticas de convivência social e que também são orientadas por suas aspirações e afetos ${ }^{17}$.

O Brasil e outros países em desenvolvimento passaram, nas últimas décadas, por uma transição nutricional em que o padrão alimentar baseado no consumo de cereais, leguminosas, raízes e tubérculos vem sendo substituído por uma alimentação rica em gorduras e açúcares, com aumento no consumo de proteínas de origem animal, e lipídios de origem animal e vegetal. Essas mudanças nos padrões de consumo associadas a fatores como hereditariedade, obesidade e inatividade física tem contribuído para a elevação na prevalência da hipertensão e do diabetes na população ${ }^{19}{ }^{20}$.

Entre os idosos estudados, observou-se que a prática de um consumo alimentar adequado vai muito além de querer, tal ideia inclui vários fatores relevantes como comodidade, necessidade, fatores emocionais, falta de condições financeira, questões familiares entre outros, dependendo individualmente de cada realidade. E mesmo diante dos resultados, pode-se analisar que tais idosos possuem alguns hábitos alimentares que poderiam ser melhorados durante os tratamentos. Eles possuem um alto consumo de alimentos ultraprocessados, sendo um fator de risco para as $\mathrm{DCNT}^{21}$, como a HAS. Apesar do resultado aparecer em semanalmente, a maior porcentagem, em relação às proteínas animais, se observa relacionada a um alto consumo de carne diante dos relatos dos pacientes "intercalam para não enjoar ou alguns tipos faz mal comer todos os dias.

Alguns estudos mostram que o aumento da relação entre a gordura poliinsaturada e a saturada em uma dieta contendo aproximadamente $25 \%$ de lipídios encontra-se associado com diminuição da pressão arterial, enquanto outros não encontram relação consistente entre quantidade e tipo de gordura ingerida e essa doença 2022 .

Por outro lado, o consumo regular de alimentos in natura, que inclue frutas e hortaliças, teve uma elevada prevalência entre os entrevistados. $\mathrm{O}$ aumento do consumo de frutas e hortaliças é uma das metas do Plano de Ações Estratégicas para o enfrentamento das DCNT no Brasil ${ }^{7}$. A importância de hortaliças e frutas na dieta de indivíduos hipertensos em diversos estudos com adultos e idosos, também evidenciaque a ingestão adequada de frutas e hortaliças está associada com a prevenção de DCV pela combinação benéfica de micronutrientes presentes em sua composição ${ }^{23}$.

\section{Conclusão}

Os resultados desse estudo mostram que grande parte dos idosos hipertensos não possuem hábitos alimentares e de vida saudáveis, além de não praticarem atividade física regular. Por isso, sugere-se um acompanhamento nutricional como parte da política pública da entrega de medicamentos no município avaliado, que vise melhorar a qualidade de vida dessa parcela da população, a fim de prevenir doenças cardiovasculares e garantir, assim como é estabelecido na Constituição Federal do Brasil de 1988, "a redução do risco de doença e de outros agravos e ao acesso universal e igualitário às ações e serviços para sua promoção, proteção e recuperação".

Além disso, observa-se a necessidade de proporcionar práticas de educação alimentar e nutricional em todos os estágios de vida, através de oficinas que estimulem a adoção de comportamentos saudáveis, como o cuidado com alimentação, controle do estresse e a prática de atividade física. Essas práticas podem impactar, positivamente, a qualidade de vida do idoso. 


\section{Referências Bibliográficas}

1. Ferreira OGL, Maciel SC, Costa SMG, et al. Envelhecimento Ativo e Sua Relação Com a Independência Funcional. Texto e Context Enferm 2012; 21: 513-518.

2. Pereira R, Cotta R, Frabceschini S. Fatores associados ao estado nutricional no envelhecimento. Rev Médica Minas Gerais 2006; 16: 160-164.

3. Wachholz PA, Rodrigues SC, Yamane R. Estado nutricional e a qualidade de vida em homens idosos vivendo em instituição de longa permanência em Curitiba, PR. Rev Bras Geriatr e Gerontol 2011; 14: 625-635.

4. Fontes AP, Botelho MA, Fernandes AA. A funcionalidade dos mais idosos ( $\geq 75$ anos): conceitos, perfis e oportunidades de um grupo heterogêneo. Rev. Bras. Geriatr. Gerontol., Rio de Janeiro, 2013; 16: 91-107.

5. Nascimento Costa F. Comparação do estado nutricional, qualidade de vida e capacidade funcional entre idosos institucionalizados e não institucionalizados. Univ Estadual Paul "Júlio Mesquita Filho"- Fac Med 2017; 1-22.

6. Mariath AB, Grillo LP, Da Silva RO, et al. Obesidade e fatores de risco para o desenvolvimento de doenças crônicas não transmissíveis entre usuários de unidade de alimentação e nutrição. Cad Saude Publica 2007; 23: 897-905.

7. Saúde M Da. Ministério da Saúde Brasília-DF 2011. 2011.

8. BRASIL. Guia alimentar para a população Brasileira. Brasília - DF, 2014. Epub ahead of print 2014. DOI: 10.1007/978-3-540-72604-3_154.

9. Barbetta PA. Cap 03 - Técnicas de amostragem. Estatística Apl às ciências sociais.

10. Franco FS. Sintomas depressivos em idosos com síndrome metabólica na Estratégia Saúde da Família de Viçosa - MG. Diss Mestr em Ciência da Nutr - Univ Fed Viçosa 2012; 100.

11. Lilliefors, H.W. On the Kolmogorov-Smirnov Test for Normality with Mean and Variance Unknown. Jour. Of the American Statistical Association, 2012, 62, 399-402

12. Mcknight, P.E., Najab, J. Teste U de Mann-Whitney. Wiley Online Library, 2010

13. Girden, E.R. ANOVA: Repeated measures, 1992, 84, 127-129.

14. Souza AO De, Mirian Ueda Yamaguchi. Adesão e não adesão dos idosos ao tratamento anti-hipertensivo. Saúde e Pesqui 2015; 8: 113-122.

15. Ribeiro AG, Minardi R, Cotta M, et al. Representações sociais de mulheres portatdoras de hipertensão arterial sobre sua enfermidade : desatando os nós da lacuna da adesão ao tratamento na agenda da Saúde da Família. Rev Saúde Coletiva 2010; 21: 87-112.

16. Ribeiro AG, Cotta RMM, Ribeiro SMR. A promoção da saúde e a prevenção integrada dos fatores de risco para doenças cardiovasculares. Cienc e Saude Coletiva 2012; 17 : 7-17.

17. Lima MT, Bucher JSNF, Lima JW de O. A hipertensão arterial sob o olhar de uma população carente: estudo exploratório a partir dos conhecimentos, atitudes e práticas. Cad Saude Publica 2004; 20: 1079-1087.

18. Zaitune MP do A, Barros MB de A, Lima MG, et al. Fatores associados ao tabagismo em idosos: Inquérito de saúde no estado de São Paulo (ISA-SP). Cad Saude Publica 2012; 28: 583-595.

19. Rinaldi AEM, Pereira AF, Macedo CS, et al. Contribuições das práticas alimentares e inatividade física para o excesso de peso infantil. Rev Paul Pediatr 2008; 26: 271-277.

20. Martins M do PSC, Gomes ALM, Martins M do C de C e, et al. Consumo alimentar, pressão arterial e controle metabólico em idosos diabéticos hipertensos. Rev Bras Cardiol 2010; 23: 162-170.

21. Martins P de FA, Faria LRC. Alimentos ultraprocessados: uma questão de saúde pública. Comun em Ciências da Saúde 2018; 29: 14-17.

22. Cassani R., Casella Filho A, Fenelon G, et al. I Diretriz sobre o consumo de gorduras e 
saúde cardiovascular. Arq Bras Cardiol 2013; 100: 1-40.

23. Silva SV. Hipertensão e seus fatores associados em idosos da cidade de Natal/RN. Rev Científica da Esc da Saúde - Catussaba 2016; Ano 5: 105-119.

\section{Como citar este artigo:}

Vieira LL, Freitas LA, Dutra LV, Araujo TA, Oliveira IM. Associação entre hábitos alimentares e de vida de idosos hipertensos usuários de uma farmácia municipal. Rev. Aten. Saúde. 2021; 19(70): 201-210. 